Z Rheumatol 2010 - 69:678-679

DOI 10.1007/s00393-009-0581-9

Online publiziert: 24. September 2010

(c) Springer-Verlag 2010

\author{
M. Schneider ${ }^{1}$ • U. Müller-Ladner ${ }^{2}$ \\ ${ }^{1}$ Klinik für Endokrinologie, Diabetologie und Rheumatologie, \\ Heinrich-Heine-Universität Düsseldorf \\ 2 Abteilung für Rheumatologie und Klinische Immunologie, Kerckhoff-Klinik, \\ Lehrstuhl für Innere Medizin mit Schwerpunkt Rheumatologie, \\ Justus-Liebig-Universität Gießen, Bad Nauheim
}

\title{
Kardiovaskuläre Komplikationen und Therapiestrategien
}

alle Patienten mit chronisch-entzündlichen Arthritiden ein jährliches Screening bezüglich kardiovaskuläre Risikofaktoren erhalten.

Effekten. Glücklicherweise haben wir Rheumatologen gute Möglichkeiten und Erfolge, Schmerzen zu lindern, und jetzt auch, entzündlich-rheumatische Erkrankungen in Remission zu bringen. Unsere wirkliche Aufgabe besteht jedoch darin, langfristig Prävention zu betreiben, um Menschen mit Rheuma vor weiterem Schaden zu schützen, der möglicherweise weit über denjenigen am muskuloskelettalen System hinausgeht.

Auch für Patienten mit rheumatischen Erkrankungen stellen kardiovaskuläre Krankheiten die häufigste Todesursache dar. Das vorliegende Schwerpunktheft beschäftigt sich praxisnah mit den Interaktionen zwischen Rheuma, Atherosklerose und therapeutischen Interventionen. Ziel dabei ist, die aktuelle Datenlage offen zu legen und Mythen zu beseitigen, die eine angemessene Therapie verhindern.

Fischer-Betz et al. geben einleitend in ihrem Beitrag Entzündungsbedingte kardiovaskuläre Morbidität: Pathophysiologie und Therapie einen Überblick über die Bedeutung und die Konsequenzen kardiovaskulärer Komorbidität bei rheumatischen Erkrankungen. Auf Basis des aktuellen Konzepts zur Pathophysiologie der Atherosklerose diskutieren sie den Einfluss klassischer Therapieformen und traditioneller Risikofaktoren. Sie unterstützen die Empfehlungen der EULAR (European League against Rheumatism), dass

\section{() Für Patienten mit rheumatischen Erkrankungen stellen kardiovaskuläre Krankheiten die häufigste Todesursache dar}

Vordenbäumen et al. gehen in ihrem Artikel Therapie der Dyslipidämie bei entzündlich-rheumatischen Erkrankungen daran anschließend gezielt auf den Risikofaktor Dyslipidämie ein. In diesem Beitrag wird das Ineinandergreifen von Rheuma und Atherosklerose besonders an der Therapiestrategie deutlich, die aus einer effektiven Krankheitskontrolle, Lebensstiländerungen und medikamentöser Cholesterinsenkung besteht. Nur so können ein ungünstiges Lipidprofil und das Mortalitätsrisiko gesenkt werden. Die Autoren machen dabei auf einen großen Auftrag an uns als Rheumatologen aufmerksam: Trotz der erhöhten kardiovaskulären Morbidität wird die Dyslipidämie bei Patienten mit entzündlich-rheumatischen Erkrankungen bisher nur selten gezielt behandelt.

Legt man Daten aus den USA zu SLE (systemischer Lupus erythematodes) zugrunde, liegt eine Ursache wohl auch in der Angst vor Nebenwirkungen durch Statine - sowohl bei Ärzten als auch bei
Patienten. Auf eine diese Komplikationen gehen Fleck et al. in ihrem Beitrag Lipidstoffwechsel aktuell: Statininduzierte Myopathien ein. Sie machen deutlich, dass Einfluss auf das Risiko einer statininduzierten Myopathie, insbesondere den Wechselwirkungen mit anderen Medikamenten, die Statindosierung, die Charakteristika des eingesetzten Statinpräparates und Patientenfaktoren wie Komorbidität, Alter und Geschlecht haben. Die Klinik der Myopathie mit typischerweiser symmetrischen, proximal betonten Muskelschmerzen, die bei körperlicher Belastung zunehmen, ist wenig richtungweisend. Die Autoren geben jedoch konkrete Empfehlungen, wie im Verdachtsfall mit einer statininduzierten Myopathie umzugehen ist.

Einen weiteren, zunehmend wichtiger werdenden Aspekt der Gesamtthematik beleuchten Tarner et al. in ihrem Artikel Biologika und kardiovaskuläres Risiko. Sie gehen insbesondere auf die Themen Herzinsuffizienz und -infarkt ein. Bei den zahlreichen Komorbiditäten, die unsere RAPatienten (RA: rheumatoide Arthritis), die regelhaft auf Biologika eingestellt werden (müssen), bereits haben, sind Fragen zu kardialen Risiken bei jedem 3.-4. Patienten zu diskutieren. Die Autoren zeigen auf, wie spärlich die Datenlage zu den einzelnen Themen ist, da kontrollierte Studien dazu aufgrund der kurzen Laufzeiten meist keine Aussage machen können. Trotzdem gibt uns auch dieser Artikel klare Empfehlungen. 
Dies trifft sicher auch auf den wohl brisantesten Beitrag von Herrn Brune mit dem Titel Kardiovaskuläre Komplikationen mit Zyklooxygenasehemmern: Mythen und Fakten zu. Er legt noch einmal dar, dass alle Hemmer der Zyklooxygenasen, selektive genauso wie nichtselektive, mit einem erhöhten kardiovaskulären Risiko einhergehen. So genannte COX-2-Hemmer (Zyklooxygenase-2-Hmmer) sind richtig dosiert nicht gefährlicher sind als traditionelle NSAR (nichtsteroidalen Antirheumatika). Es geht also - so Brune - darum, die Anwendung der Zyklooxygenasehemmer zu verbessern, und er gibt dazu sehr gute Anregungen.

Auch wenn mit den von uns gewählten Beiträgen sicher nur einige wesentliche Teile dieser klinisch relevanten Thematik angesprochen werden, macht bereits diese Auswahl deutlich, wie komplex dieser Bereich ist - und dies für jeden einzelnen Betroffenen. Fasst man die gemachten Angaben nur grob abschätzend zusammen, können wir das therapeutische Konzept in Bezug auf kardiovaskuläre Komplikationen wohl bei jedem 2. Rheumakranken optimieren. Das ist die erste Aufgabe. Die zweite ergibt sich aus der Tatsache, dass mit Ausnahme der Daten zu den Coxiben alle anderen noch bestehenden Fragen nicht durch randomisiert-kontrollierte Studien beantwortet werden können. Mehr und bessere Daten erhalten wir nur aus Langzeitbeobachtungen, d. h. Registern. Deshalb besteht eine weitere Schlüsselaufgabe für die Rheumatologen in Deutschland darin, möglichst alle Patienten in RABBIT (Rheumatoide Arthritis: Beobachtung der Biologikatherapie) zu dokumentieren. Damit leisten wir einen wesentlichen Beitrag zu Optimierung der Versorgung unserer eigenen Patienten.

Ihre

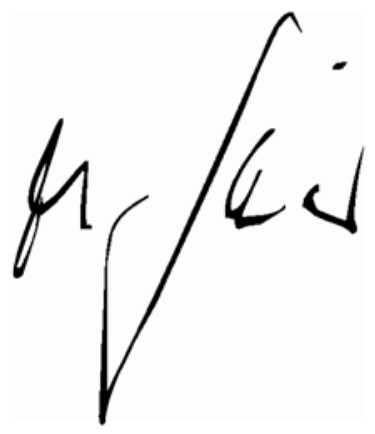

Matthias Schneider

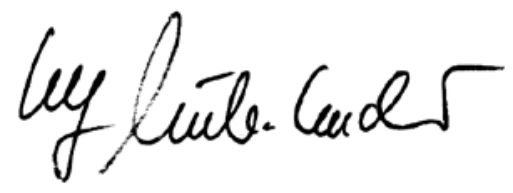

Ulf Müller-Ladner

\section{Korrespondenzadresse}

Prof. Dr. M. Schneider

Klinik für Endokrinologie,

Diabetologie und Rheumatologie, Heinrich-Heine-Universität Düsseldorf Moorenstraße 5, 40225 Düsseldorf schneiderm@med.uni-duesseldorf.de

\section{Erfolgreicher Leitlinienkongress in Chicago}

Vom 26. bis 28. August 2010 fand in Chicago der 7. Kongress des Guidelines International Network (G-I-N) statt.

G-I-N ist ein Zusammenschluss international führender Institutionen auf dem Gebiet der Entwicklung medizinischer Leitlinien. Die „Non-for-Profit-Organisation" wurde 2002 gegründet mit dem Ziel, die Qualität medizinischer Versorgung weltweit zu verbessern. Unter dem Motto „Von der besten Evidenz zur besten Versorgung" fand in Chicago ein intensiver Wissenstransfer zwischen führenden Leitlinienexperten aller Kontinente statt. Diskussionen zu den aktuellen Herausforderungen evidenzbasierter Medizin standen dabei im Mittelpunkt. Fünf hochkarätig besetzte Plenumsdiskussionen beleuchteten Fragen wie „Medizin, Medien und Politik", ,Interessenkonflikte", "Rationierung medizinischer Leistungen" und "Patientenbeteiligung an der Entwicklung medizinischer Leitlinien“. Während der Tagung wurde auch der neue Vorstand gewählt: Mit Philipp van der Wees von der Königlich Niederländischen Gesellschaft für Physiotherapie ist erstmalig kein Arzt erster Vorsitzender des internationalen Leitliniennetzwerks. Mit dieser Entscheidung dokumentiert G-I-N, dass moderne medizinische Leitlinien unter Beteiligung aller wichtigen Fach- und Berufsgruppen im Gesundheitswesen entwickelt werden müssen, wenn sie angemessene und umsetzbare Antworten auf die komplexen Anforderungen der Versorgung geben sollen.

Quelle:

Guidelines International Network (G-I-N), c/o AEZQ, www.g-i-n.net 